DMD \# 45930

\title{
Drug Metabolism and Disposition
}

Brain distribution and bioavailability of elacridar after different routes of administration in the mouse.

\section{Ramola Sane, Sagar Agarwal, William F. Elmquist}

Department of Pharmaceutics, Brain Barriers Research Center, University of Minnesota, Minneapolis, MN, USA 
DMD \#45930

Running Title: Elacridar brain distribution and bioavailability

\section{Corresponding Author:}

William F. Elmquist, Pharm. D, PhD., Professor and Department Head, Department of

Pharmaceutics, University of Minnesota, 308 Harvard Street SE, Minneapolis, MN 55455,

Phone: 612-625-0097, Email: elmqu011@umn.edu

\section{Manuscript statistics:}

Number of figures: 4

Number of tables: 2

Number of Pages: 30

Number of References: 26

Number of Words in Abstract: 223

Number of words in Introduction: 740

Number of words in Discussion: 1,630

\section{List of abbreviations:}

BBB, blood-brain barrier; CNS, central nervous system; P-gp, p-glycoprotein; BCRP, breast cancer resistance protein; FVB, Friend Leukemia Virus Strain B; LC-MS/MS, liquid chromatography-tandem mass spectrometry; HPMC, hydroxyl propyl methyl cellulose; DMSO,

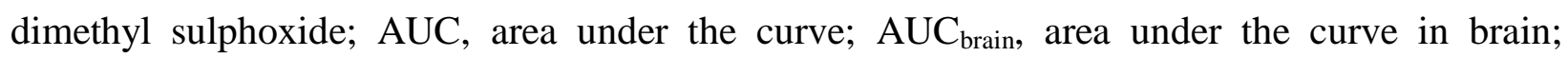
$\mathrm{AUC}_{\text {plasma, }}$ area under the curve in plasma; $\mathrm{AUC}_{\text {extrapolated, }} \mathrm{AUC}$ from last measured time point to infinity; $\mathrm{AUC}_{\mathrm{inf}}, \mathrm{AUC}$ from time zero to infinity; TKI, tyrosine kinase inhibitor; IP, intraperitoneal; IV, intravenous; PO, per os 
DMD \#45930

\section{Abstract:}

The objective of this study was to determine the bioavailability and disposition of elacridar (GF120918) in plasma and brain after various routes of administration in the mouse. Elacridar is a potent inhibitor of P-gp and BCRP and has been used to examine the influence of these efflux transporters on drug distribution to brain. FVB mice were administered $100 \mathrm{mg} / \mathrm{kg}$ elacridar either orally or intraperitoneally. The absolute bioavailability of elacridar after oral or intraperitoneal dosing was determined with respect to an intravenous dose of $2.5 \mathrm{mg} / \mathrm{kg}$. At these doses, the absolute bioavailability was 0.22 for oral administration and 0.01 for intraperitoneal administration. The terminal half-life of elacridar was approximately 4 hours after intraperitoneal and intravenous administration, and nearly 20 hours after oral dosing. The brain-to-plasma partition coefficient (Kp,brain) of elacridar increased as plasma exposure increased, suggesting saturation of the efflux transporters at the blood-brain barrier. The Kp,brain after intravenous, intraperitoneal, and oral dosing was $0.82,0.43$ and 4.31, respectively. The low aqueous solubility and high lipophilicity of elacridar result in poor oral absorption, most likely dissolution-rate limited. These results illustrate the importance of the route of administration and the resultant plasma exposure in achieving effective plasma and brain concentrations of elacridar, and can be used as a guide for future studies involving elacridar administration, and in developing formulation strategies to overcome the poor absorption. 
DMD \#45930

\section{Introduction:}

Elacridar (GF 120918) is a potent inhibitor of p-glycoprotein (P-gp) and breast cancer resistance protein (BCRP) (Witherspoon et al., 1996; Allen et al., 1999). It is a third generation inhibitor (Tan et al., 2000) and was initially described as a multi-drug resistance reversal agent (Hyafil et al., 1993), where it restored the sensitivity of multidrug resistant tumors to doxorubicin.

Elacridar has been used extensively in vitro and in vivo as a P-gp and BCRP inhibitor. When coadministered with P-gp substrates such as topotecan and paclitaxel, elacridar improved their oral absorption by inhibiting intestinal P-gp thereby preventing efflux of substrate drugs into the intestinal lumen (Kruijtzer et al., 2002; Bardelmeijer et al., 2004). The role of P-gp and BCRP in limiting the distribution of substrate drugs across the blood-brain barrier has been examined using elacridar as a dual inhibitor of both P-gp and BCRP. Co-administration of elacridar improved the brain penetration of several substrate molecules, such as morphine and amprenavir (Letrent et al., 1998; Edwards et al., 2002). Elacridar also significantly increased brain distribution of several tyrosine kinase inhibitors (TKIs) including imatinib, dasatinib, gefitinib, sorafenib, and sunitinib (Bihorel et al., 2007; Chen et al., 2009; Lagas et al., 2009; Agarwal et al., 2010; Lagas et al., 2010; Agarwal et al., 2011b; Tang et al., 2012a). More recently, it was shown that brain penetration of the tyrosine kinase inhibitor sunitinib and its active metabolite is limited by the P-gp and BCRP at the blood-brain barrier. The oral administration of elacridar improved the brain penetration of sunitinib in wild-type mice by 12 - fold such that it was equal to that seen in Mdrla/b(-/-)Bcrpl(-/-) mice (Tang et al., 2012a; Tang et al., 2012b). Elacridar has also been administered to glioma xenograft bearing mice to enhance the brain penetration of paclitaxel (Hubensack et al., 2008). 
DMD \#45930

The common objective of many of the above studies was to use P-gp and Bcrp inhibition by elacridar as a strategy to enhance distribution of substrate drugs into the brain. One such therapeutic area where this could be particularly useful is in the treatment of devastating brain tumors such as glioma. Many molecularly targeted tyrosine kinase inhibitors that are currently being evaluated in glioma do not effectively cross an intact BBB due to P-gp- and Bcrpmediated efflux (Breedveld et al., 2005; Bihorel et al., 2007; Chen et al., 2009; Lagas et al., 2009). Co-administration of elacridar with TKIs that do not effectively cross the BBB due to Pgp and BCRP mediated efflux could lead to improved efficacy of these drugs in glioma as a result of their enhanced delivery across the blood-brain barrier. Since these TKIs are intended for chronic administration, for elacridar to effectively improve delivery, it must also be administered chronically. To accomplish this efficiently, we must have a better understanding of the factors that could affect the systemic bioavailability and brain distribution of elacridar.

Chronic administration of elacridar has several difficulties, mainly arising due to its unfavorable physicochemical properties. Elacridar is practically insoluble in water, poorly soluble in most other aqueous solvents and it is extremely lipophilic ( $\log \mathrm{P}=5.67)$ (Padowski and Pollack, 2010). This makes it difficult to formulate elacridar as an injectable. These poor physicochemical properties also mean that its oral absorption will most likely be dissolution-rate limited (Ward and Azzarano, 2004). This will also contribute to observed variability in variability in plasma and tissue concentrations in preclinical studies. Intersubject variability in exposure following oral dosing has been observed in clinical trials (Planting et al., 2005). Brain penetration of elacridar in mice has been shown to be dose-dependent and influenced by the presence of $\mathrm{P}$-gp and BCRP at the BBB. This has been elegantly demonstrated using radiolabelled elacridar PET imaging (Kawamura et al., 2011a; Kawamura et al., 2011b). All of 
DMD \#45930

these factors contribute to a very variable adsorption and disposition of elacridar in both preclinical models and clinical applications.

While elacridar has been used to alter the brain penetration of a wide variety of drugs, the factors influencing the brain distribution of elacridar itself have not been carefully elucidated. The objective of this study was to describe the pharmacokinetics of elacridar in plasma and brain after different routes of administration and to estimate the systemic bioavailability of elacridar.

The results from the current study will be helpful in determining the dose and route of administration of elacridar in future studies that may involve chronic administration, particularly in preclinical studies using the mouse model. 
DMD \#45930

\section{Materials and Methods}

Materials :Elacridar ( GF 120918 ) [N-(4-(2-(1,2,3,4-tetrahydro-6,7-dimethoxy-2-

isoquinolinyl)ethyl)phenyl)-9,10-dihydro-5-methoxy-9-oxo-4-acridine carboxamide],molecular weight 563.64, was purchased from Toronto Research Chemicals (Toronto, Ontario, Canada). Hydroxypropylmethylcellulose (Methocel E5LV) was obtained from Dow Chemical Company (Midland, MI). All other chemicals used were reagent grade or HPLC grade from Sigma-Aldrich (St. Louis, MO).

Animals: In vivo studies were conducted in FVB wild-type mice (Taconic Farms, Germantown, NY). All animals were 8-10 weeks old at the time of the experiment. All mice were maintained under a 12 hour light/dark cycle and had unlimited access to food and water and were maintained under a temperature controlled environment. All studies were approved by the Institutional Animal Care and Use Committee of the University of Minnesota.

Intravenous administration of elacridar: The elacridar intravenous dosing solution was prepared on the day of the experiment by dissolving elacridar in a vehicle containing DMSO, propylene glycol and saline $(2: 2: 1 \mathrm{v} / \mathrm{v} / \mathrm{v})$ at a concentration of $1.25 \mathrm{mg} / \mathrm{ml}$. FVB wild-type mice were injected with an intravenous dose of $2.5 \mathrm{mg} / \mathrm{kg}(2 \mu \mathrm{l}$ volume/g of bodyweight $)$ into the tail vein. Blood and brain were collected at $0.5,1,2,4$ and 8 hours, post dose $(n=4$ at each time point). Animals were euthanized by use of a carbon dioxide chamber. Blood was collected by cardiac puncture and plasma was obtained by centrifugation at $7500 \mathrm{rpm}$ for $10 \mathrm{~min}$ at $4{ }^{\circ} \mathrm{C}$. The whole brain was quickly removed from skull and rinsed with ice-cold saline. Brains were 
DMD \#45930

immediately flash frozen with liquid nitrogen. The specimens were stored at $-80^{\circ} \mathrm{C}$ until analysis by LCMS-MS.

Intraperitoneal and oral administration of elacridar: Elacridar for intraperitoneal and oral dosing was prepared on the day of the experiment by preparing a stable suspension of elacridar, using $0.5 \%$ HPMC and $1 \%$ Tween 80 to obtain a $10 \mathrm{mg} / \mathrm{ml}$ formulation. Mice received an intraperitoneal dose of $100 \mathrm{mg} / \mathrm{kg}$ by injection into the peritoneal cavity. For oral administration, mice received a dose of $100 \mathrm{mg} / \mathrm{kg}$ by oral gavage. Blood and brain were sampled at $15 \mathrm{~min}, 0.5$, 1, 2, 4, 8 hours after intraperitoneal dosing and at $0.5,1,2,4,8,17$ and 24 hours after oral dosing. Plasma and brain samples were collected and processed in the manner described above.

Analysis of elacridar by LCMS-MS: The concentrations of elacridar in mouse plasma and brain were determined by HPLC coupled with mass spectrometry. Frozen brain samples were thawed and homogenized with three volumes of $5 \%$ bovine serum albumin using a tissue homogenizer (Thermo Fisher Scientific, Waltham MA). $50 \mu 1$ of plasma and $100 \mu 1$ of brain homogenate were spiked with $20 \mathrm{ng}$ of internal standard, tryphostin (AG 1478) and $100 \mu \mathrm{l}$ of a pH 11 buffer ( $0.1 \mathrm{M}$ sodium hydroxide and 0.04 M sodium bicarbonate). Samples were extracted by vigorously vortexing with $1 \mathrm{ml}$ of ethyl acetate for $5 \mathrm{~min}$ and then centrifugation at $7500 \mathrm{rpm}$ for $15 \mathrm{~min}$ at $4^{\circ} \mathrm{C} .600 \mu \mathrm{l}$ of organic layer was transferred to microcentrifuge tubes and dried a gentle stream of nitrogen. Samples were reconstituted in $100 \mu 1$ of mobile phase and transferred to autosampler vials. A $5 \mu \mathrm{l}$ volume was injected using a temperature controlled autosampler maintained at $10^{\circ} \mathrm{C}$. Chromatographic analysis was performed using an Agilent Technologies (Santa Clara, CA) Eclipse XDB-C18 RRHT threaded column (4.6 mm id X 12.5 $\mathrm{mm}, 5 \mu$ ). The mobile phase was composed of acetonitrile: $20 \mathrm{mM}$ ammonium formate (with 0.1 $\%$ formic acid) $(42: 58 \mathrm{v} / \mathrm{v})$ with a flow rate of $0.25 \mathrm{ml} / \mathrm{min}$. The eluent was monitored using a 
DMD \#45930

Thermo Finnigan TSQ Quantum 1.5 detector (Thermo Fisher Scientific, Waltham, MA). The instrument was equipped with an electrospray interface. The samples were ionized by the electrospray probe and analyzed in the positive ionization mode operating at a spray voltage of $4500 \mathrm{~V}$ for both elacridar and the internal standard. The spectrometer was programmed to allow the $[\mathrm{MH}]+$ ion of elacridar at m/z 564.6 and that of internal standard at 316.67 to pass through the first quadrupole (Q1) and into the collision cells (Q2). The collision energy was set at $39 \mathrm{~V}$ for elacridar and 9V for tryphostin. The product ions for elacridar (m/z 252.9) and internal standard (m/z 300.9) were monitored through quadrupole 3(Q3). The scan width and scan time for monitoring the two product ions were $1.5 \mathrm{~m} / \mathrm{z}$ and $0.5 \mathrm{~s}$, respectively. The assay was precise and linear over a range of $2.5 \mathrm{ng} / \mathrm{ml}$ to $1500 \mathrm{ng} / \mathrm{ml}$ (Coefficient of variation was less than $10 \%$ over all concentrations).

Pharmacokinetic calculations: Pharmacokinetic parameters from the concentration-time profile in plasma and brain were calculated by non-compartmental analysis using Phoenix WinNonlin 6.1 (Mountain View, CA). The terminal rate constants were determined using the last three data points for plasma and brain. The areas under concentration-time curve for plasma $\left(\mathrm{AUC}_{\mathrm{plasma}}\right)$ and brain $\left(\mathrm{AUC}_{\text {brain }}\right)$ from time 0 to infinity were calculated using the linear trapezoidal method with the $\mathrm{AUC}_{\text {extrapolated }}$ (AUC from last measured time point to infinity) estimated by dividing the last measured concentration by the elimination rate constant. After intravenous dosing, the concentration at time zero $\left(\mathrm{C}_{0}\right)$ was back-extrapolated by log-linear regression of first two data points. The $\mathrm{AUC}_{\text {plasma }}$ from time zero to infinity after intravenous injection was then determined as above. The absolute bioavailability after oral and intraperitoneal administration was calculated as, 
DMD \#45930

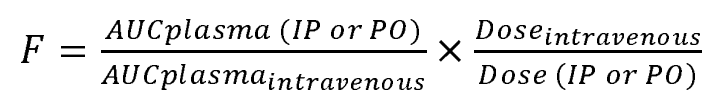

The brain-to-plasma partition coefficient of elacridar (Kp, brain) after different routes of administration was calculated as a ratio of $\mathrm{AUCs}\left(\mathrm{AUC}_{\mathrm{brain}} / \mathrm{AUC}_{\mathrm{plasma}}\right)$. The brain-to-plasma concentration ratio at each time point was calculated as a ratio of brain concentration to plasma concentration $\left(\mathrm{C}_{\text {brain }} / \mathrm{C}_{\text {plasma }}\right)$.

\section{Statistical Analysis:}

SigmaPlot for Windows version 11.0 (Systat Software, Inc., San Jose, CA) was used to determine if a difference between two groups was statistically significant. One-way analysis of variance with the Holm-Sidak post hoc test was used for multiple comparisons at a significance level of 0.05 . 
DMD \#45930

\section{Results:}

Intravenous administration of elacridar: The disposition of elacridar in plasma and brain was studied in FVB wild-type mice following an intravenous injection. The plasma concentrations showed a bi-exponential decline indicating distinct distribution and elimination phases (Figure 1a). Concentrations rapidly reached peak levels in brain within 0.5 hours after intravenous dosing with the maximum concentration $\left(\mathrm{C}_{\max }\right)$ observed at 0.5 hours, the first measured time point. The brain-to-plasma concentration ratio was high at the initial time points (up to 2 hours) and decreased thereafter as concentrations in brain declined more rapidly compared to those in plasma (Figure 1b). This is consistent with the observed terminal half-life of 4.4 hours in plasma and 1.5 hours in brain (Table 1). The total plasma clearance was estimated to be $0.46 \mathrm{ml} / \mathrm{min}$. The blood flow to the liver for a $20 \mathrm{~g}$ mouse is $1.8 \mathrm{ml} / \mathrm{min}$ (Davies and Morris, 1993), meaning elacridar has at most a low to moderate hepatic extraction in the mouse. The $\mathrm{AUC}_{0 \text {-inf }}$ was 161 $\mu \mathrm{g}^{*} \mathrm{~min} / \mathrm{ml}$ in plasma and $131 \mu \mathrm{g} * \mathrm{~min} / \mathrm{ml}$ in brain. The resulting brain Kp ratio was 0.82 , indicating that after intravenous dosing at $2.5 \mathrm{mg} / \mathrm{kg}$ there is approximately equal partitioning of elacridar into the brain as plasma.

Intraperitoneal administration of elacridar: The plasma and brain concentrations after an intraperitoneal dose of $100 \mathrm{mg} / \mathrm{kg}$ were measured in FVB wild type mice. After intraperitoneal dosing, brain concentrations were significantly lower than plasma concentrations at all measured time-points, except at 4 hours post dose (Figure 2a). The corresponding brain-to-plasma concentration ratios remained less than one at all measured time points (Figure 2b). It is important to note that the plasma concentrations after intravenous administration at which we observe a greater than one brain-to-plasma concentration ratio are higher than the maximum plasma concentrations seen following intraperitoneal dosing. The brain distribution ratio (i.e., the 
DMD \#45930

partition coefficient) of elacridar is likely to be dependent on its plasma levels. The observed $\mathrm{C}_{\max }$ in plasma after the intraperitoneal dose was $0.295 \pm 0.06 \mu \mathrm{g} / \mathrm{ml}$ and that in brain was 0.061 $\pm 0.024 \mu \mathrm{g} / \mathrm{ml}$. The apparent plasma clearance $(\mathrm{Cl} / \mathrm{F})$ was estimated to be $33 \mathrm{ml} / \mathrm{min}$ by noncompartmental analysis. The $\mathrm{AUC}_{0 \text {-inf }}$ in plasma was $90.3 \mu \mathrm{g}^{*} \mathrm{~min} / \mathrm{ml}$ and $43.5 \mu \mathrm{g}^{*} \mathrm{~min} / \mathrm{ml}$ in the brain. The elimination phase half-life of elacridar after non compartmental analysis was estimated to be 4.3 hours in plasma and 9.2 hours in brain. The Kp ratio was 0.48 , indicating that the partitioning into the brain was lower after intraperitoneal administration, even at a dose 40 times higher, as compared to intravenous administration.

Oral administration of elacridar: The brain and plasma pharmacokinetics of elacridar were studied in FVB wild-type mice after a $100 \mathrm{mg} / \mathrm{kg}$ dose administered orally. The brain concentrations were lower than plasma concentrations until 1 hour post dose, after which the brain concentrations were several fold higher than plasma concentrations (Figure 3a). The brain to plasma ratio after oral administration was less than one for initial time points, and then increased reaching a maximum of approximately 6 at 4 hours post-dose, before showing a slow decline (Figure 3b). Non-compartmental analysis of plasma and brain concentration-time data showed that the plasma $\mathrm{AUC}_{0 \text {-inf }}$ was $1460 \mu \mathrm{g} * \mathrm{~min} / \mathrm{ml}$ and brain $\mathrm{AUC}_{0 \text {-inf }}$ was $6296 \mu \mathrm{g} * \mathrm{~min} / \mathrm{ml}$ (Table 1). The $\mathrm{C}_{\max }$ in brain after oral dose was $4.34 \pm 0.79 \mu \mathrm{g} / \mathrm{ml}$, significantly higher than that seen after intraperitoneal dosing. The time to reach $\mathrm{C}_{\max }$ in plasma was 4 hours, indicating a slow dissolution and absorption from the gut. Post oral dosing, the brain Kp was found to be 4.31, suggesting that a high dose of elacridar administered orally gives high distribution in the brain. The half-life of the drug in the brain after the oral dose mirrored its plasma half-life, with the values being 19.8 and 15.6 hours, respectively. 
DMD \#45930

\section{Determination of bioavailability after intraperitoneal and oral administration: Absolute}

bioavailability was determined as a ratio of dose normalized AUC after intraperitoneal or oral administration to dose normalized AUC after IV administration (Table 2). The bioavailability after intraperitoneal administration was $1.3 \%$ and that after oral administration was $22 \%$ with the suspension. The bioavailability after intraperitoneal dosing was very poor, indicating that intraperitoneal may not a favorable route of administration to get reproducible plasma and brain exposure of elacridar using this simple suspension formulation. The bioavailability of elacridar after oral administration was higher than after an equivalent dose administered intraperitoneally, this could be due to enhanced dissolution in the gut through greater solvent availability or micellar effects bile salts. The half-life of elacridar after oral administration was approximately 5 times longer than after IV or IP administration. There is a possibility that there is non-linearity in the absorption, distribution and possibly elimination of elacridar. However, the bioavailability has been calculated with the assumption that there is no change in the clearance of the drug with increase in plasma exposure of the drug. 
DMD \#45930

\section{DISCUSSION}

Elacridar is a third generation inhibitor of the transporters P-gp and BCRP (Hyafil et al., 1993; Witherspoon et al., 1996). It has been used to determine the influence of P-gp and BCRP on the brain distribution of drugs that are substrates for P-gp and BCRP (Breedveld et al., 2005; Bihorel et al., 2007; Chen et al., 2009; Agarwal et al., 2010; Agarwal et al., 2011b). It has also been suggested as a tool to improve the efficacy of drugs such as sunitinib in the treatment of glioma (Tang et al., 2012a). The objective of this study was to investigate the brain and plasma pharmacokinetics of elacridar in the mouse, and determine its bioavailability after oral and intraperitoneal administration.

The physicochemical properties of elacridar such as poor solubility and high lipophilicity could be responsible for the variability that is observed in plasma concentrations in this study. This inter-individual variability has been previously observed before in humans upon oral administration of elacridar (Kuppens et al., 2007) and was attributed to variability in dissolution of elacridar. In a study done in mice, the rate of dissolution of elacridar was also found to be a factor in limiting the plasma exposure of orally administered elacridar, despite increasing its dose (Ward and Azzarano, 2004).

Oral dosing is a convenient route for drug administration in both rodents and humans, especially for chronic administration. Recently, it was shown that brain penetration of the tyrosine kinase inhibitor sunitinib, and its active metabolite is limited by P-gp and BCRP at the blood brain barrier. The oral administration of elacridar at a $100 \mathrm{mg} / \mathrm{kg}$ dose improved the brain penetration of sunitinib in wild-type mice by nearly 12- fold (Tang et al., 2012a; Tang et al., 2012b). Both plasma and brain exposure of elacridar after a $100 \mathrm{mg} / \mathrm{kg}$ oral dose were significantly higher than 
DMD \#45930

after intraperitoneal administration. The partition coefficient of elacridar in the brain after oral administration was 4.31, indicating good distribution of elacridar from systemic circulation into the brain following this oral dose. Interestingly, the elimination half-life after an oral dose of 100 $\mathrm{mg} / \mathrm{kg}$ was close to 20 hours, approximately 5 -fold greater than the half-lives after intraperitoneal or intravenous dosing. There could be several possible reasons for this phenomenon. The increased oral half-life could be due to a slow dissolution rate (given the poor aqueous solubility) which limits the rate of absorption and thus leads to an extended release period. So the observed terminal half-life might be a measure of a possible long absorption halflife (flip-flop kinetics). The finding that the observed Tmax in plasma was 8 hours post dose supports this hypothesis. Another reason for the extended half-life could be a change in hepatic or renal clearance of the drug due to the higher plasma exposures of elacridar. However, elacridar is not a potent inhibitor of any P450 enzymes in vitro (Ward and Azzarano, 2004), with the $\mathrm{IC}_{50} \mathrm{~s}$ in micromolar range. The $\mathrm{AUC}_{\text {plasma }}$ (dose normalized) was greater following oral dosing versus intraperitoneal dosing, which resulted in a greater bioavailability. This may be explained by inhibition of intestinal P-gp by elacridar after its oral administration, which would also improve its absorption from the gastro-intestinal tract, contributing to a higher bioavailability as compared to intraperitoneal administration. Another possible explanation of this phenomenon could be enterohepatic recycling of elacridar upon oral administration, which could lead to a larger than expected area under the curve. Irrespective of these possibilities, the results show that high dose administration of elacridar by the oral route can yield high exposures in both plasma and brain. Tang et al., suggested that a $100 \mathrm{mg} / \mathrm{kg}$ dose could represent an effective dose in mice and indicated that there is no significant toxicity associated with administration of this dose (Tang et al., 2012a). Although toxicity was not evaluated in the 
DMD \#45930

current study, there were no overt elacridar related adverse effects after this single dose in mice. However, if a chronic dosing regimen is to be considered, the concerns for toxicity cannot be dismissed, especially considering the high plasma concentrations and the extended half-life of elacridar (that could lead to significant accumulation at steady state) that are seen with this dose.

Intraperitoneal dosing is also an attractive option for chronic dosing, especially in preclinical studies. The mice that received elacridar intraperitoneally, showed a much lower plasma exposure as compared to oral administration after an equivalent dose. This may most likely be due to poor dissolution and/or absorption of the drug from the peritoneal cavity. The plasma and brain concentrations also showed significant inter-animal variability. The intraperitoneal route of administration, while convenient to dose chronically in mice, resulted in a reduced plasma exposure of elacridar, therefore, the applications of an intraperitoneal dosing regimen with the current suspension formulation are limited. In the current study, the $\mathrm{Kp}\left(\mathrm{AUC}_{\mathrm{brain}} / \mathrm{AUC}_{\mathrm{plasma}}\right)$ ratio after an intraperitoneal dose of $100 \mathrm{mg} / \mathrm{kg}$ was 0.48 . In a similar study by Padowski and Pollack (Padowski and Pollack, 2010), an intraperitoneal dose of $10 \mathrm{mg} / \mathrm{kg}$ yielded a Kp of 0.0784. Taken together, these findings may indicate the presence of a non-linearity in plasma and brain exposures with increasing dose, especially after intraperitoneal dosing.

Exposure after an intravenous dose was used as the reference for calculating the absolute bioavailability after the other two routes of administration. After an intravenous dose of 2.5 $\mathrm{mg} / \mathrm{kg}$, the AUC in plasma and brain of elacridar were approximately equal, yielding a Kp ratio of 0.82 (Table 1). Despite observing this substantial brain distribution of elacridar after intravenous administration, intravenous dosing is not a viable option for chronic dosing in noncatheterized mice due to difficulties in performing repeated injections. Moreover, the dosing solution prepared for intravenous administration is unstable because it is prone to precipitation. 
DMD \#45930

The bioavailability of elacridar is limited by its poor physicochemical properties. The bioavailability of elacridar after oral administration of a high dose was about $22 \%$, while that after intraperitoneal administration at the same dose was only about $1 \%$. The higher $\mathrm{AUC}_{\text {plasma }}$ after oral administration versus the intraperitoneal administration could be due to the solubilizing effect of bile salts in the gut. Oral administration of elacridar, when using this simple suspension formulation, appears to be the most effective way to achieve plasma exposures necessary to effectively inhibit P-gp and BCRP at the BBB.

There were significant findings in this study related to the brain penetration of elacridar after the different routes of administration. First, the $\mathrm{Kp}$ for elacridar (a measure of its brain distribution) was found to be dependent on its plasma exposure (see Figure 4). When the plasma exposure was relatively high, as seen after oral and intravenous administration, the Kp ratio was greater than one, with the highest Kp ratio of $\sim 5$ seen after oral dosing, which yielded the highest plasma exposure. The Kp ratio after the intraperitoneal route of administration, that resulted in a relatively low plasma exposure, was less than one. Second, the brain-to-plasma concentration ratios plotted as a function of time for all routes of administration showed an increase to a maximum value followed by a decrease (see Figures $1 b, 2 b, 3 b$ ). This was unexpected because after reaching a steady-state in the tissue of distribution (pseudo distributional equilibrium), the brain-to-plasma concentration ratio should remain constant. One explanation for both these findings can be the active efflux of elacridar from the brain by P-gp and BCRP. Elacridar inhibits both these transporters at the $\mathrm{BBB}$ and it is possible that the mechanism behind the inhibitory action might be competitive (due to it being a substrate for the two transporters). This has been shown to be true in a study that used PET imaging and transporter knockout mice to study the influence of P-gp and BCRP on elacridar distribution at the BBB (Kawamura et al., 2011a; 
DMD \#45930

Kawamura et al., 2011b). The above finding is currently being further investigated in another study. However, it does explain why brain exposures are dependent on plasma concentrations. As the plasma concentrations drop below levels that are required to saturate efflux at the BBB, the net efflux from the brain will become greater than the passive diffusion into the brain. This would result in the brain concentrations decreasing more rapidly than the corresponding plasma concentration, ultimately resulting in a decreasing trend in brain-to-plasma ratios with respect to time.

Concurrent administration of elacridar with drugs that are substrates for P-gp and BCRP improves their distribution across the BBB, and could lead to improved efficacy. The study of elacridar pharmacokinetics in the brain is important if we consider the issue of target cells that are present behind an intact $\mathrm{BBB}$ in the invasive rim of a brain tumor or the normal brain (Agarwal et al., 2011a). Distribution of elacridar in the brain tissue could possibly address the issue of target cells that express BCRP and P-gp and are therefore resistant to chemotherapy (Lu and Shervington, 2008). Elacridar in the brain could be effective in inhibiting P-gp and BCRP present on these cells, allowing the chemotherapeutic agents to act on them, possibly preventing recurrence of the tumor. A targeted approach for CNS delivery that employs elacridar could also reduce the dose required to achieve effective brain concentrations thus reducing systemic toxicity.

The use of elacridar has been limited in both preclinical and clinical situations partly due its poor solubility and poor bioavailability. It is an unmet need to improve the bioavailability and solubility of elacridar. There are several possible methods that could be explored, including synthesis of water soluble prodrugs, preparation of solid dispersions, use of surfactants, cyclodextrins, and permeation enhancers. 
DMD \#45930

In summary, this study has examined the pharmacokinetics of elacridar in plasma and brain in mice and determined its bioavailability after different routes of administration. The results from these experiments can be used to help guide the selection of doses and routes of administration of elacridar for future studies. 
DMD \#45930

\section{Authorship Contributions}

Participated in research design: Sane, Elmquist

Conducted experiments: Sane, Agarwal

Contributed new reagents or analytic tools: Sane, Elmquist

Performed data analysis: Sane, Elmquist

Wrote or contributed to the writing of the manuscript: Sane, Agarwal, Elmquist 
DMD \#45930

\section{References:}

Agarwal S, Sane R, Gallardo JL, Ohlfest JR and Elmquist WF (2010) Distribution of gefitinib to the brain is limited by P-glycoprotein (ABCB1) and breast cancer resistance protein (ABCG2)-mediated active efflux. J Pharmacol Exp Ther 334:147-155.

Agarwal S, Sane R, Oberoi R, Ohlfest JR and Elmquist WF (2011a) Delivery of molecularly targeted therapy to malignant glioma, a disease of the whole brain. Expert Rev Mol Med 13:e17.

Agarwal S, Sane R, Ohlfest JR and Elmquist WF (2011b) The role of the breast cancer resistance protein $(\mathrm{ABCG})$ in the distribution of sorafenib to the brain. J Pharmacol Exp Ther 336:223-233.

Allen JD, Brinkhuis RF, Wijnholds J and Schinkel AH (1999) The mouse Bcrp1/Mxr/Abcp gene: amplification and overexpression in cell lines selected for resistance to topotecan, mitoxantrone, or doxorubicin. Cancer Res 59:4237-4241.

Bardelmeijer HA, Ouwehand M, Beijnen JH, Schellens JH and van Tellingen O (2004) Efficacy of novel P-glycoprotein inhibitors to increase the oral uptake of paclitaxel in mice. Invest New Drugs 22:219-229.

Bihorel S, Camenisch G, Lemaire M and Scherrmann JM (2007) Influence of breast cancer resistance protein $(\mathrm{Abcg} 2)$ and p-glycoprotein $(\mathrm{Abcb} 1 \mathrm{a})$ on the transport of imatinib mesylate (Gleevec) across the mouse blood-brain barrier. J Neurochem 102:1749-1757.

Breedveld P, Pluim D, Cipriani G, Wielinga P, van Tellingen O, Schinkel AH and Schellens JH (2005) The effect of Bcrp1 (Abcg2) on the in vivo pharmacokinetics and brain penetration of imatinib mesylate (Gleevec): implications for the use of breast cancer 
DMD \#45930

resistance protein and P-glycoprotein inhibitors to enable the brain penetration of imatinib in patients. Cancer Res 65:2577-2582.

Chen Y, Agarwal S, Shaik NM, Chen C, Yang Z and Elmquist WF (2009) P-glycoprotein and breast cancer resistance protein influence brain distribution of dasatinib. J Pharmacol Exp Ther 330:956-963.

Davies B and Morris T (1993) Physiological parameters in laboratory animals and humans. Pharm Res 10:1093-1095.

Edwards JE, Brouwer KR and McNamara PJ (2002) GF120918, a P-glycoprotein modulator, increases the concentration of unbound amprenavir in the central nervous system in rats. Antimicrob Agents Chemother 46:2284-2286.

Hubensack M, Muller C, Hocherl P, Fellner S, Spruss T, Bernhardt G and Buschauer A (2008) Effect of the ABCB1 modulators elacridar and tariquidar on the distribution of paclitaxel in nude mice. J Cancer Res Clin Oncol 134:597-607.

Hyafil F, Vergely C, Du Vignaud P and Grand-Perret T (1993) In vitro and in vivo reversal of multidrug resistance by GF120918, an acridonecarboxamide derivative. Cancer Res 53:4595-4602.

Kawamura K, Yamasaki T, Konno F, Yui J, Hatori A, Yanamoto K, Wakizaka H, Ogawa M, Yoshida Y, Nengaki N, Fukumura T and Zhang MR (2011a) Synthesis and in vivo evaluation of (18)F-fluoroethyl GF120918 and XR9576 as positron emission tomography probes for assessing the function of drug efflux transporters. Bioorg Med Chem 19:861870.

Kawamura K, Yamasaki T, Konno F, Yui J, Hatori A, Yanamoto K, Wakizaka H, Takei M, Kimura Y, Fukumura T and Zhang MR (2011b) Evaluation of limiting brain penetration 
DMD \#45930

related to P-glycoprotein and breast cancer resistance protein using [(11)C]GF120918 by PET in mice. Mol Imaging Biol 13:152-160.

Kruijtzer CM, Beijnen JH, Rosing H, ten Bokkel Huinink WW, Schot M, Jewell RC, Paul EM and Schellens JH (2002) Increased oral bioavailability of topotecan in combination with the breast cancer resistance protein and P-glycoprotein inhibitor GF120918. J Clin Oncol 20:2943-2950.

Kuppens IE, Witteveen EO, Jewell RC, Radema SA, Paul EM, Mangum SG, Beijnen JH, Voest EE and Schellens JH (2007) A phase I, randomized, open-label, parallel-cohort, dosefinding study of elacridar (GF120918) and oral topotecan in cancer patients. Clin Cancer $\operatorname{Res}$ 13:3276-3285.

Lagas JS, van Waterschoot RA, Sparidans RW, Wagenaar E, Beijnen JH and Schinkel AH (2010) Breast cancer resistance protein and P-glycoprotein limit sorafenib brain accumulation. Mol Cancer Ther 9:319-326.

Lagas JS, van Waterschoot RA, van Tilburg VA, Hillebrand MJ, Lankheet N, Rosing H, Beijnen JH and Schinkel AH (2009) Brain accumulation of dasatinib is restricted by Pglycoprotein $(\mathrm{ABCB} 1)$ and breast cancer resistance protein $(\mathrm{ABCG} 2)$ and can be enhanced by elacridar treatment. Clin Cancer Res 15:2344-2351.

Letrent SP, Pollack GM, Brouwer KR and Brouwer KL (1998) Effect of GF120918, a potent Pglycoprotein inhibitor, on morphine pharmacokinetics and pharmacodynamics in the rat. Pharm Res 15:599-605.

Lu C and Shervington A (2008) Chemoresistance in gliomas. Mol Cell Biochem 312:71-80. 
DMD \#45930

Padowski JM and Pollack GM (2010) Examination of the ability of the nasal administration route to confer a brain exposure advantage for three chemical inhibitors of $\mathrm{P}$ glycoprotein. J Pharm Sci 99:3226-3233.

Planting AS, Sonneveld P, van der Gaast A, Sparreboom A, van der Burg ME, Luyten GP, de Leeuw K, de Boer-Dennert M, Wissel PS, Jewell RC, Paul EM, Purvis NB, Jr. and Verweij J (2005) A phase I and pharmacologic study of the MDR converter GF120918 in combination with doxorubicin in patients with advanced solid tumors. Cancer Chemother Pharmacol 55:91-99.

Tan B, Piwnica-Worms D and Ratner L (2000) Multidrug resistance transporters and modulation. Curr Opin Oncol 12:450-458.

Tang SC, Lagas JS, Lankheet NA, Poller B, Hillebrand MJ, Rosing H, Beijnen JH and Schinkel $\mathrm{AH}$ (2012a) Brain accumulation of sunitinib is restricted by P-glycoprotein (ABCB1) and breast cancer resistance protein (ABCG2) and can be enhanced by oral elacridar and sunitinib coadministration. Int J Cancer 130:223-233.

Tang SC, Lankheet NA, Poller B, Wagenaar E, Beijnen JH and Schinkel AH (2012b) Pglycoprotein $(\mathrm{ABCB} 1)$ and Breast Cancer Resistance Protein (ABCG2) restrict brain accumulation of the active sunitinib metabolite $\mathrm{N}$-desethyl sunitinib. J Pharmacol Exp Ther. 341(1):164-73.

Ward KW and Azzarano LM (2004) Preclinical pharmacokinetic properties of the Pglycoprotein inhibitor GF120918A (HCl salt of GF120918, 9,10-dihydro-5-methoxy-9oxo-N-[4-[2-(1,2,3,4-tetrahydro-6,7-dimethoxy-2-i soquinolinyl)ethyl]phenyl]-4acridine-carboxamide) in the mouse, rat, dog, and monkey. J Pharmacol Exp Ther 310:703-709. 
DMD \#45930

Witherspoon SM, Emerson DL, Kerr BM, Lloyd TL, Dalton WS and Wissel PS (1996) Flow cytometric assay of modulation of P-glycoprotein function in whole blood by the multidrug resistance inhibitor GG918. Clin Cancer Res 2:7-12. 
DMD \#45930

\section{Footnotes:}

This work was supported by National Institutes of Health - National Cancer Institute

[CA138437] (W.F.E.) and a Faculty Development grant at the University of Minnesota (W.F.E,).

Financial support for Sagar Agarwal was provided by the Doctoral Dissertation Fellowship from

the University of Minnesota. Financial support for Ramola Sane was provided by the Ronald J.

Sawchuk Fellowship and Rowell Fellowship. 
DMD \#45930

\section{Figure legends:}

Figure 1a: Elacridar concentrations in plasma and brain after a single intravenous dose of 2.5 $\mathrm{mg} / \mathrm{kg}$ in FVB wild-type mice. Brain concentrations are higher at corresponding higher plasma concentration and decrease as plasma concentrations decrease. ${ }^{*}, p<0.05$. Mean \pm S.D. $(n=4$ at each time point)

Figure 1b: Brain-to-plasma concentration ratio for elacridar after a single intravenous dose of $2.5 \mathrm{mg} / \mathrm{kg}$ in FVB wild-type mice. The brain-to-plasma concentration ratio is greater than one at initial time points and then drops below unity as plasma concentration decrease. Data presented as mean \pm S.D. $(n=4$ at each time point $)$

Figure 2a: Elacridar concentrations in plasma and brain after a single intraperitoneal dose of 100 $\mathrm{mg} / \mathrm{kg}$ in FVB wild-type mice. Brain concentrations are significantly lower than plasma concentrations indicating that brain delivery of elacridar after intraperitoneal administration is limited. $* \mathrm{p}<0.05$. Mean $\pm \mathrm{SD}(\mathrm{n}=4$ at each time point $)$

Figure 2B: Brain-to-plasma concentration ratio for elacridar after a single intraperitoneal dose of $100 \mathrm{mg} / \mathrm{kg}$ in FVB wild-type mice. The brain-to-plasma concentration ratio is less than unity at all time-points indicating poor brain distribution. Data presented as mean \pm S.D. $(n=4$ at each time point)

Figure 3a: Elacridar concentrations in plasma and brain after a single oral dose of $100 \mathrm{mg} / \mathrm{kg}$ in FVB wild-type mice. Brain concentrations are significantly lower than plasma concentrations at early time points and higher than plasma at later time points. ${ }^{*}, \mathrm{p}<0.05$. Mean $\pm \operatorname{SD}(\mathrm{n}=4$ at each time point) 
DMD \#45930

Figure 3b: Brain-to-plasma concentration ratios for elacridar after a single oral dose of 100 $\mathrm{mg} / \mathrm{kg}$ in FVB wild-type mice. The brain-to-plasma concentration ratio is less than unity at initial times, but increases with time. Data presented as mean \pm S.D. ( $n=4$ at each time point).

Figure 4: Kp ratio (AUCbrain/AUCplasma) of elacridar after different routes of administration as a function of the AUCplasma. The brain penetration of elacridar in mice is a function of the plasma AUC; as the plasma AUC increases, the brain partition coefficient of elacridar increases to greater than 1, indicating possible saturation of efflux at the BBB. 
DMD \#45930

\section{Tables:}

Table 1: Plasma and brain pharmacokinetic parameters calculated by noncompartmental analysis after administration of a single dose of elacridar in FVB wild-type mice.

\begin{tabular}{|c|c|c|c|c|c|c|c|}
\hline & & $\begin{array}{l}\text { Half } \\
\text { life } \\
\text { (hr) }\end{array}$ & $\begin{array}{l}\text { Cmax } \\
(\mu \mathrm{g} / \mathrm{ml})\end{array}$ & $\begin{array}{c}\text { Tmax } \\
\text { (hr) }\end{array}$ & $\begin{array}{l}\text { AUC 0-last } \\
\left(\mu g^{*} \min / \mathrm{ml}\right)\end{array}$ & $\begin{array}{c}\text { AUC 0-inf } \\
\left(\mu g^{*} \min / \mathrm{ml}\right)\end{array}$ & Kp \\
\hline IV & Plasma & 4.4 & $0.564 \pm 0.14$ & 0.5 & $138.6 \pm 6.5$ & 161.4 & \multirow{2}{*}{0.82} \\
\hline $2.5 \mathrm{mg} / \mathrm{kg}$ & Brain & 1.5 & $1.05 \pm 0.38$ & 0.5 & $128.3 \pm 19.6$ & 131.3 & \\
\hline IP & Plasma & 4.3 & $0.295 \pm 0.06$ & 0.5 & $63.5 \pm 6.6$ & 90.3 & \multirow{2}{*}{0.48} \\
\hline $100 \mathrm{mg} / \mathrm{kg}$ & Brain & 9.2 & $0.061 \pm 0.02$ & 4 & $19.9 \pm 4.7$ & 43.5 & \\
\hline $\mathrm{PO}$ & Plasma & 20 & $0.78 \pm 0.12$ & 8 & $792.8 \pm 67.9$ & 1460 & \multirow{2}{*}{4.31} \\
\hline $100 \mathrm{mg} / \mathrm{kg}$ & Brain & 16 & $4.34 \pm 0.79$ & 8 & $3887 \pm 410$ & 6296 & \\
\hline
\end{tabular}


DMD \#45930

Table 2: Plasma pharmacokinetics and bioavailability calculated by noncompartmental analysis of data from IV, IP and PO administration.

\begin{tabular}{|c|c|c|c|c|c|c|}
\hline Route & $\begin{array}{c}\text { Dose } \\
\text { (mg/kg) }\end{array}$ & $\begin{array}{c}\mathrm{CL} / \mathrm{F} \\
(\mathrm{ml} / \mathrm{min})\end{array}$ & $\begin{array}{c}\mathrm{Vd} / \mathbf{F} \\
\text { (L) }\end{array}$ & $\begin{array}{l}T_{1 / 2} \\
(h r)\end{array}$ & $\begin{array}{c}\mathrm{AUC}_{(0-\mathrm{inf})} \\
\left(\mu \mathrm{g}^{*} \min / \mathrm{ml}\right)\end{array}$ & $\mathbf{F}$ \\
\hline Oral & 100 & 2.05 & 3.5 & 20 & 1460 & 0.22 \\
\hline Intraperitoneal & 100 & 33.2 & 12.3 & 4.3 & 90.3 & 0.013 \\
\hline Intravenous & 2.5 & 0.46 & 0.17 & 4.4 & 161.4 & 1 \\
\hline
\end{tabular}




\section{FIGURE 1a}

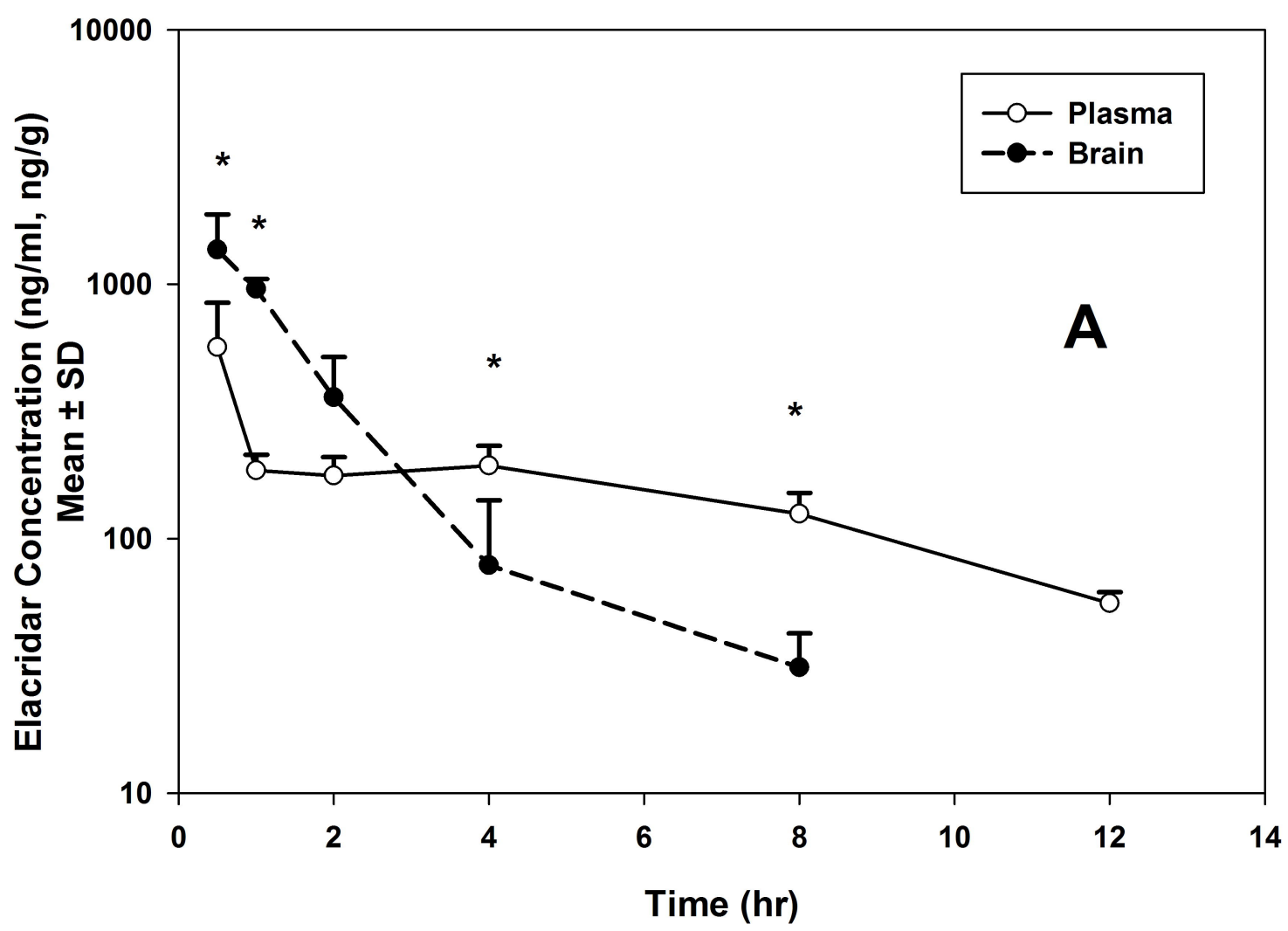


Figure 1b

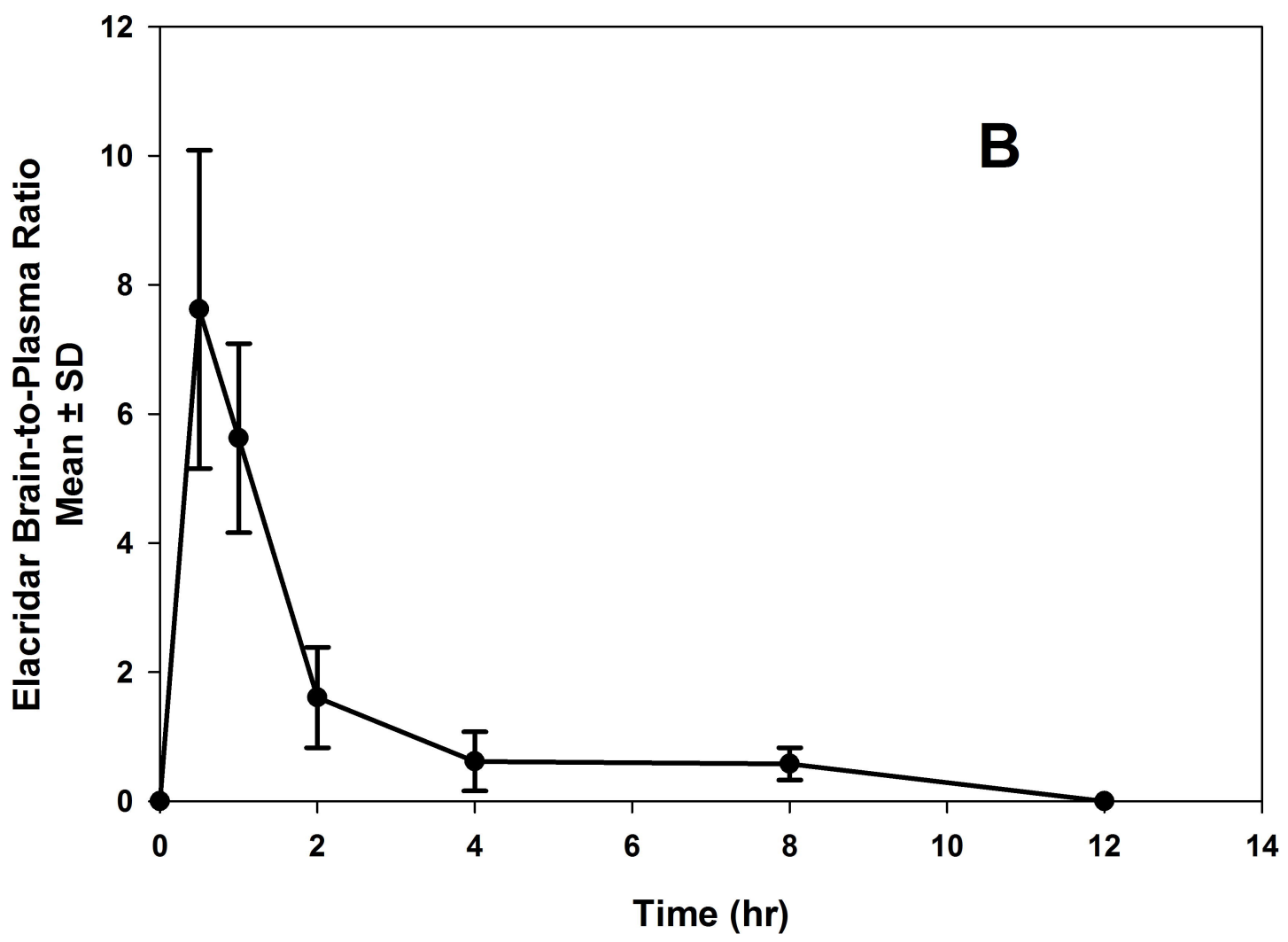


Figure 2a:

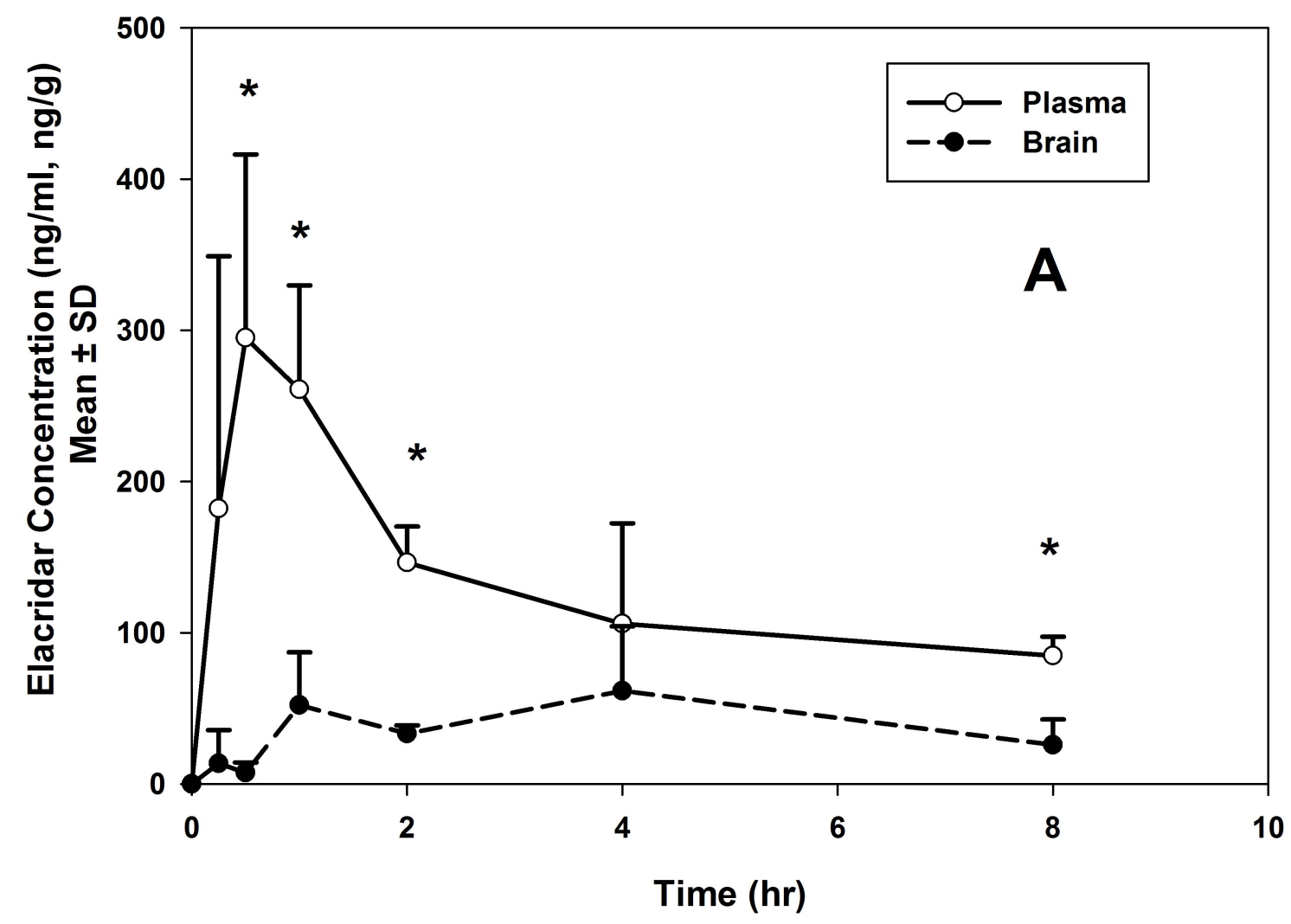


Figure 2b:

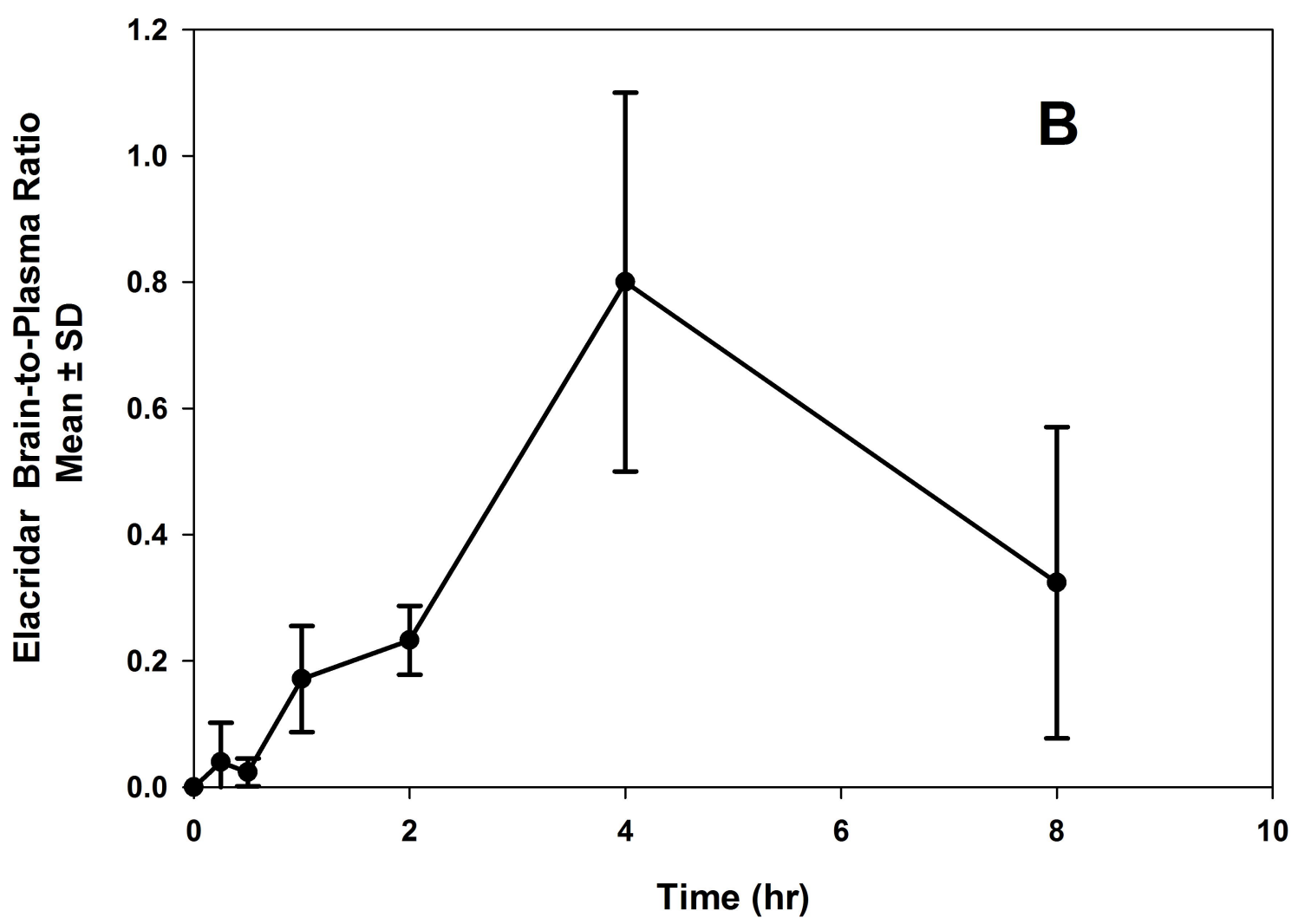


Figure 3a:

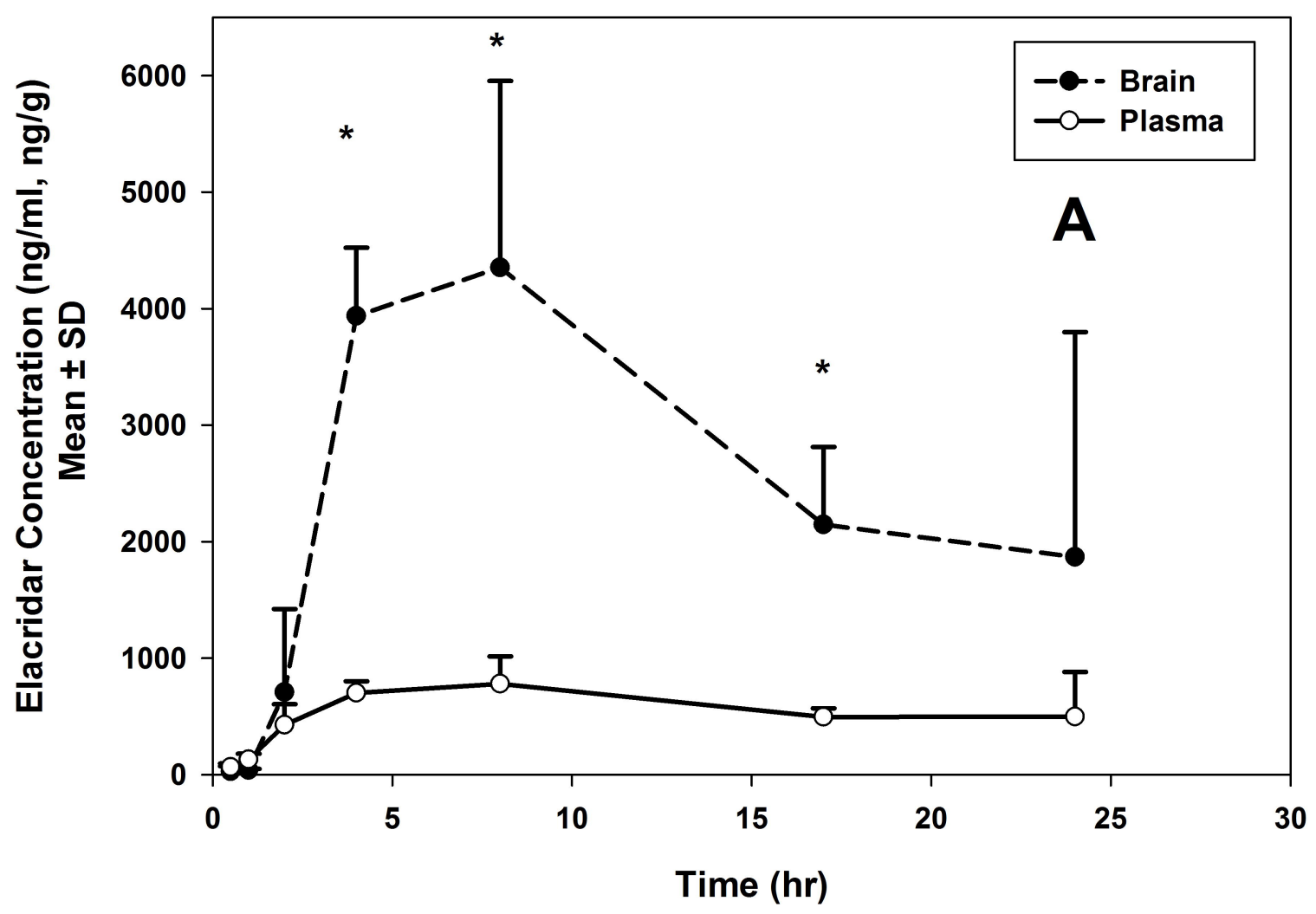


Figure 3b:

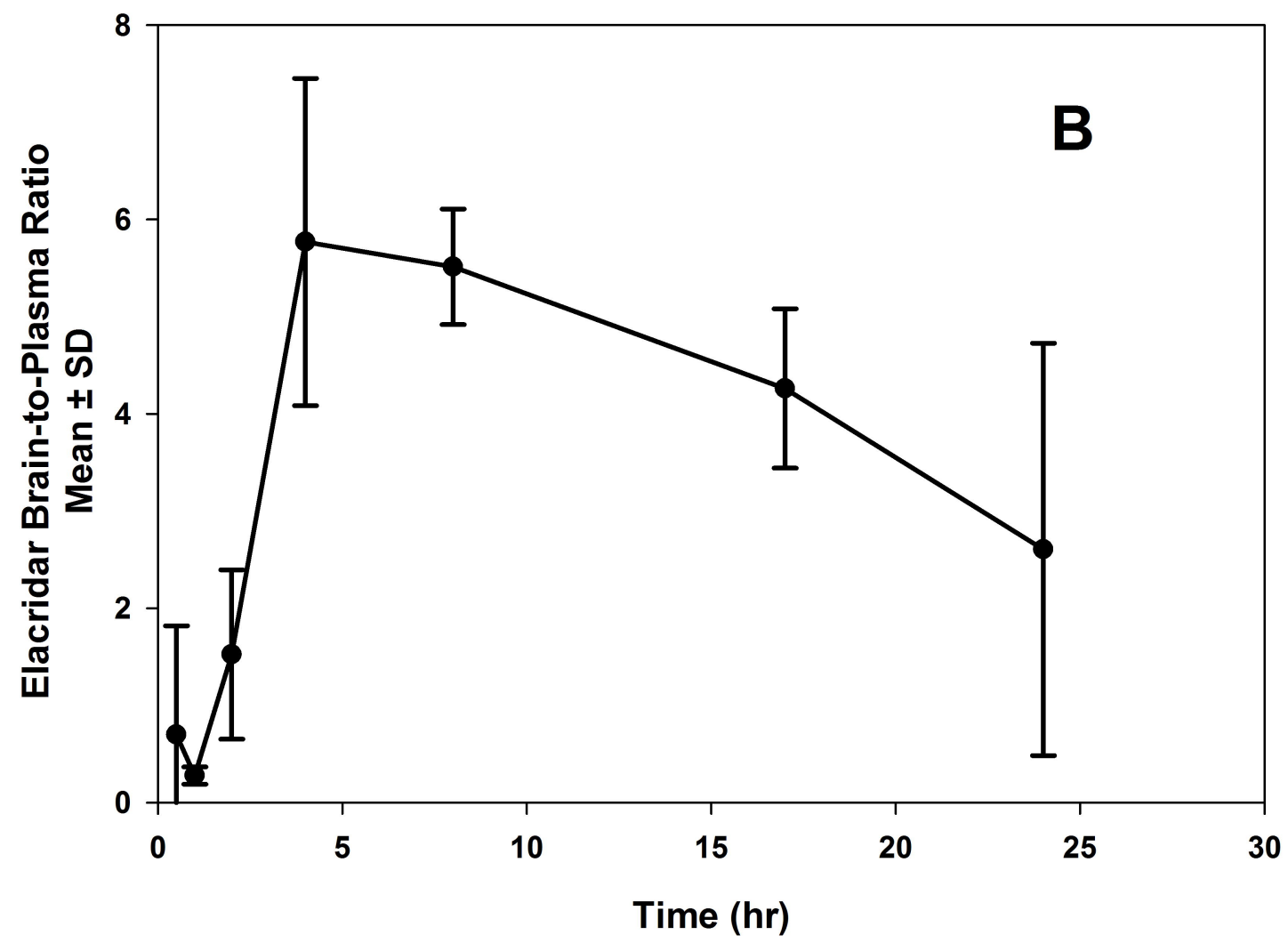


Figure 4

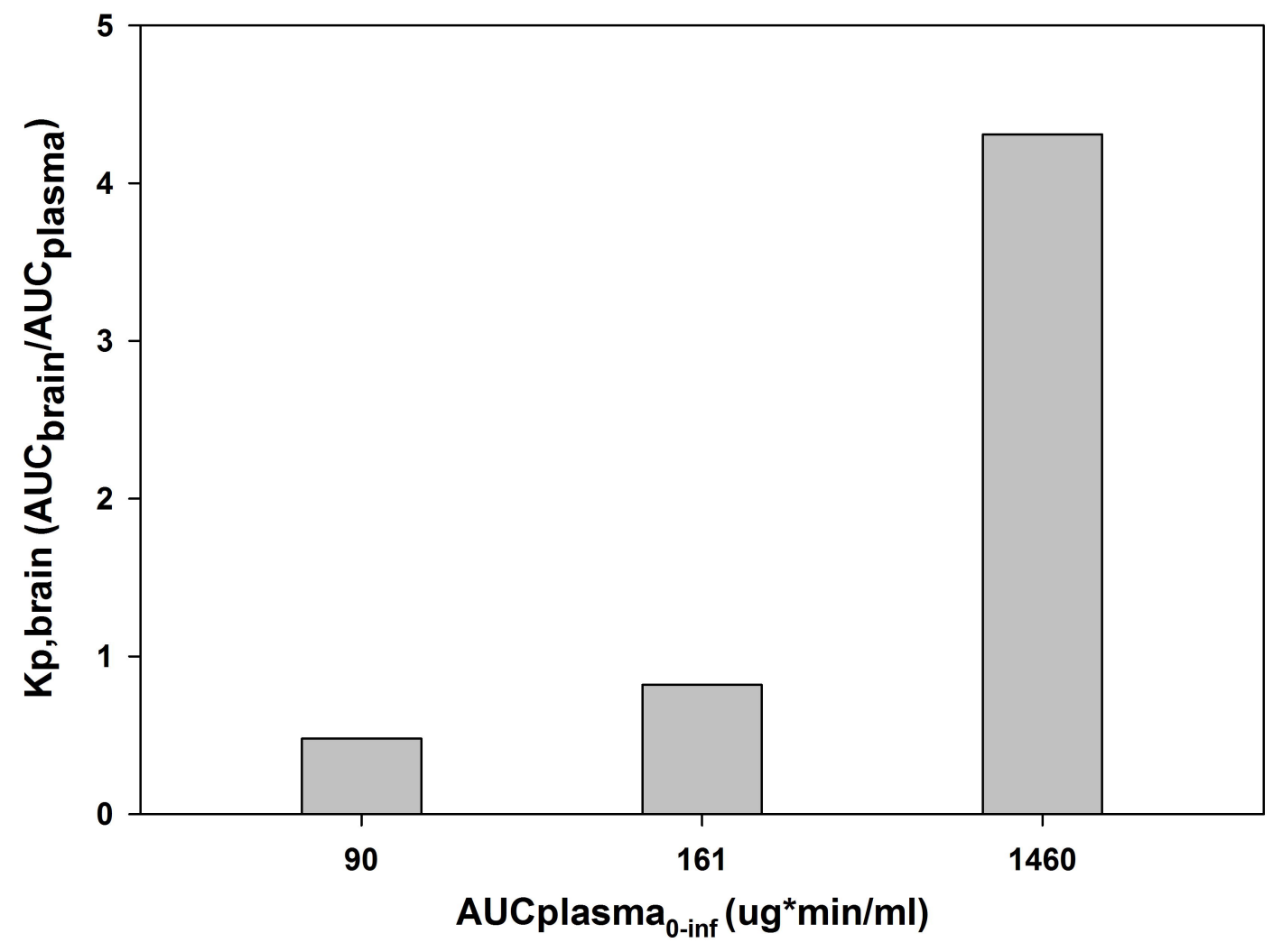

